\title{
Representaciones Sociales de la Experiencia Educativa de Jóvenes que Presentan Trastorno del Espectro Autista en Chile
}

\section{Social Representations of the Educational Experience of Young People with Autism Spectrum Disorder in Chile}

\author{
Camila Andrea Toledo Manríquez ${ }^{1 *}$ \\ Óscar Fernando Basulto Gallegos ${ }^{2}$ \\ ${ }^{1}$ Centro de Capacitación Laboral de Educación Especial Paulo Freire, Chile \\ ${ }^{2}$ Universidad Católica de la Santísima Concepción, Chile
}

\begin{abstract}
El presente artículo tiene por objetivo visibilizar representaciones sociales de la experiencia educativa de jóvenes con trastorno del espectro autista (TEA), construidas a partir de los relatos de vida de sus madres. En relación a la metodología, corresponde a un estudio cualitativo, enmarcado en un paradigma interpretativo y a su vez bajo un enfoque fenomenológico. La muestra, de tipo intencional estuvo compuesta por cinco mujeres madres de jóvenes con TEA de entre 16 y 24 años, todos con residencia en Concepción-Chile. Los relatos de vida son trabajados a partir de un análisis de contenido categorial temático con un fuerte esfuerzo hermenéutico. Los principales resultados obtenidos, se refieren a profesores de educación regular con poca preparación y disposición al trabajo con estudiantes con TEA, en contraposición a la actitud de los demás estudiantes (sin TEA), quienes, de acuerdo a los relatos, tuvieron una recepción positiva, empática y afectuosa hacia los jóvenes. Por otro lado, las madres evidencian una integración escolar que no existe y un gran temor por el futuro de sus hijos luego de los 26 años producto de la legislación vigente en Chile. A partir de los relatos surge la representación social de la discriminación y del temor, la representación social de la no integración y la representación social del futuro educativo negado, todo lo cual es explicado en el desarrollo del presente artículo.
\end{abstract}

Descriptores: Educación especial; Dificultad en el aprendizaje; Percepción; Relato de vida.

This article aims to make visible social representations of the educational experience of young people with autism spectrum disorder (ASD), built on the stories of their mothers' lives. In relation to the methodology, it corresponds to a qualitative study, framed in an interpretive paradigm and in turn under a phenomenological approach. The sample, of intentional type was composed of five women mothers of young people with ASD between 16 and 24 years, all with residence in Concepción-Chile. The life stories are worked from an analysis of thematic categorical content with a strong hermeneutical effort. The main results obtained refer to regular education teachers with little preparation and willingness to work with students with ASD, as opposed to the attitude of the other students (without ASD), who according to the stories, had a positive reception, empathic and affectionate towards young people. On the other hand, the mothers show a school integration that does not exist and a great fear for the future of their children after the age of 26 , product of the legislation in force in Chile. From the stories emerges the social representation of discrimination and fear, the social representation of non-integration and the social representation of the denied educational future, all of which is explained in the development of this article

Keywords: Special education; Difficulty in learning; Perception; Life story.

*Contacto: ctoledo@magister.ucsc.cl

ISSN: 0718-7378

www.rinace.net/rlei/
Recibido: $\quad 15 / 07 / 2019$

$1^{a}$ Evaluación: 25/09/2019

Aceptado: 04/01/2020 


\section{Introducción}

El MINEDUC (2015, p. 5) establece que el derecho a la educación, es el derecho de todos a recibir una educación de calidad en igualdad de oportunidades a lo largo de la vida, y que la inclusión es un factor fundamental de este derecho. De igual forma, señala que la diversidad es un factor que no solo favorece los procesos de aprendizaje de los estudiantes, sino que además potencia el desarrollo profesional de los docentes al tener que adecuar sus estrategias de enseñanza a las necesidades particulares de cada estudiante.

Para dar respuesta a la diversidad en el sistema educativo, Chile cuenta con un sistema de educación especial que corresponde a una modalidad que actúa de manera transversal en todos los niveles, ya sea de educación regular o especial, entregando apoyos para atender las necesidades educativas especiales de los estudiantes ya sean transitorias o permanentes (MINEDUC, 2005, p. 35). Este sistema de educación especial cuenta con la opción educativa de programa de integración escolar en escuelas regulares y la opción de escuelas especiales, opciones a las que asistieron los hijos de las participantes de la investigación.

En relación a las necesidades educativas especiales (NEE) que presentan los estudiantes, de acuerdo al decreto 170 del MINEDUC (2009), estas pueden ser de carácter transitorio o permanente. De acuerdo a dicho decreto dentro de las NEE de carácter permanente se encuentra el trastorno autista, actualmente denominado trastorno del espectro autista (TEA) según el DSM-V de la American Psychiatric Association (APA, 2013).

El decreto 170 del MINEDUC (2009, p. 22) define el TEA como:

una alteración cualitativa de un conjunto de capacidades referidas a la interacción social, la comunicación y la flexibilidad mental que pueden variar en función de la etapa del desarrollo, la edad y el nivel intelectual de la persona que lo presenta.

El TEA, se presenta con mayor frecuencia en hombres que en mujeres, en una proporción de 4:1, según datos del MINSAL (2011), y de acuerdo a la OMS (2017) tiene una prevalencia de 1 caso por cada 160 niños. En Chile, no existen bases de datos que indiquen la cantidad de personas que presentan el diagnóstico, no obstante, el SENADIS (2016) se encuentra trabajando en ello. Este dato, resultó relevante para la ejecución de este trabajo puesto que evidencia falta de conocimiento respecto del TEA en un aspecto crucial como lo es la prevalencia.

De acuerdo a Mesibov y Howly (2010) los estudiantes con TEA presentan dificultades en las áreas de comunicación, interacción social e imaginación, por lo tanto es probable que no comprendan o no interpreten el curriculum de la misma forma que el resto de los estudiantes. Bajo este panorama, los autores señalan que un sistema de educación tradicional pudiera no ser conveniente si se considera que está basado en comunicación verbal y social, áreas donde estos estudiantes presentan dificultades. Siguiendo con la educación de estudiantes con TEA, Delfos (2006) señala que la falta de conocimiento sobre el diagnóstico, provoca que la inclusión de niños y jóvenes que lo presentan en escuelas regulares sea compleja, y de acuerdo a Blanco (2006), los estudiantes se deben enfrentar a barreras de acceso, permanencia y participación.

En Chile de acuerdo a la revisión de la literatura, no existen estudios que profundicen la realidad que viven las familias de personas con TEA, como se verá más adelante, es un trastorno cuyas características lo vuelven complejo, por lo tanto la familia se ve enfrentada a una serie de emociones y cambios en su ritmo de vida. De esta forma, la falta de conocimiento en relación a la prevalencia del TEA, en relación a las vivencias de las familias 
y en relación a la inserción de quienes lo presentan en el sistema educativo, motivó la realización de esta investigación. A su vez, en este estudio se presentará más adelante la discusión teórica en que se abordarán los tópicos principales que incluyen el trastorno del espectro autista y representaciones sociales, y la construcción de dichas representaciones a través de relatos de vida.

Como ya se señaló en el resumen, el objetivo general del trabajo es visibilizar representaciones sociales de la experiencia educativa de jóvenes con TEA, construidas a partir de los relatos de vida de sus madres. Y como objetivos específicos describir las opciones educativas en que participaron los jóvenes con TEA, e identificar emociones de la experiencia educativa de los jóvenes desde los relatos de las madres.

Luego, en términos metodológicos se puede mencionar que se trabajó al alero del paradigma interpretativo y con una mirada fenomenológica, puesto que resulta vital el foco subjetivo e intersubjetivo de las madres residentes en Concepción-Chile respecto a la experiencia educativa de sus hijos con TEA, en función de la construcción de representaciones sociales.

Posteriormente, el artículo describe los principales resultados emanados de los relatos de las madres, de ellos, se puede mencionar de manera breve, que la mayoría de las madres optaron por una escuela regular para sus hijos como primera opción, pero conforme transcurrieron los años, optaron por la escuela especial, a partir de esto, se evidencia de manera explícita una concepción de la integración escolar como algo que no existe, que no resulta, una baja disposición por parte de los docentes al trabajo con los jóvenes con TEA, y como aspecto alentador, una acogida positiva por parte de los pares. De igual forma, se describen las representaciones sociales construidas en base al relato de las madres y la interpretación de los investigadores, donde se destacan las representaciones sociales de la discriminación y del temor, de la no integración, y del futuro educativo negado. Finalmente, se presentan las principales conclusiones donde emerge con fuerza la preocupación de las madres en relación al futuro educativo y ocupacional de sus hijos luego de los 26 años y, por tanto, la necesidad de ahondar en la temática en futuras investigaciones.

\section{Trastorno del espectro autista}

El concepto de autismo fue utilizado por primera vez a comienzos del siglo xx por el psiquiatra Bleuler para describir una serie de manifestaciones en pacientes con esquizofrenia (Arce, 2008). Sin embargo, la evidencia más potente del origen del diagnóstico se encuentra en la descripción que hizo Leo Kanner a 11 niños en el año 1948 en un hospital de Estados Unidos. A pesar de esta descripción realizada por Kanner, pasaron casi 40 años hasta que el autismo fue reconocido de manera oficial como un trastorno por la American Psychiatric Association (APA, 2013) en 1980 y posteriormente, fue catalogado como discapacidad por la Individuals with Disabilities Education Act (Taylor et al., 2009). Setenta años después de la primera descripción de Kanner, el Diagnostic and stadistical manual of mental disorders (DSM) de la American Psychiatric Association (APA, 2013), en su quinta versión (DSM-V), estableció la categoría diagnóstica de trastorno del espectro autista, dejando atrás el concepto utilizado en su anterior versión, trastorno generalizado del desarrollo (TGD) y además eliminó como entidades diagnósticas el trastorno autista, el síndrome Asperger, el TGD no especificado y el trastorno 
desintegrativo infantil (Borax y Buron, 2017). Además, de acuerdo al DSM-V (APA, 2013), el TEA se refiere a una dificultad persistente en el desarrollo del proceso de socialización (interacción social y comunicación social), junto con un patrón restringido de conductas e intereses, dentro de lo cual se incluyen restricciones sensoriales.

Al mismo tiempo -reforzando la idea anterior-, según Taylor y colaboradores (2009), el panorama del TEA no parece muy alentador, ya que se trata de un trastorno que afecta varias áreas de manera simultánea, por lo tanto, la escolarización de quienes lo presentan no resulta sencilla al considerar que es un estudiante que presenta dificultades serias en la comunicación, alteraciones en el lenguaje, dificultades a nivel conductual y además un coeficiente intelectual bajo. Además de lo anterior, los estudiantes presentan características sensoriales especiales, que incluyen una respuesta "anormal" frente a ciertos estímulos, por ejemplo, en algunos casos les molesta de sobre manera el ruido, texturas y sabores particulares. Por lo tanto, frente a lo anterior, el contex to educativo en la educación regular para los estudiantes que presentan TEA no es fácil, ya que se enfrentan a un ambiente colapsado de múltiples estímulos sensoriales, donde requieren comunicarse e interactuar con otros constantemente y seguir un programa de estudio estándar pese a sus dificultades cognitivas.

Para esta investigación, el relato de las madres pone en evidencia la experiencia vivida, si bien no como protagonista, si como una participante clave del proceso educativo de su hijo. Es necesario destacar que tanto los padres como la familia, se ven enfrentados a situaciones específicas al enfrentar la realidad del TEA. En relación a lo anterior, Wing (1998) señala que el niño con TEA en un comienzo es un niño aparentemente normal, por lo tanto los padres disfrutan de un hijo que parece desarrollarse como cualquier otro, hasta que comienzan a ser evidentes los primeros signos ya señalados, y otros como la falta de contacto visual y la ausencia de juego creativo. Por lo tanto, en este contexto, al recibir el diagnóstico, la familia suele vivir un duelo, debido a la sensación de pérdida de expectativa e ilusiones implantadas en este hijo (Hernández et al., 2012, p. 75), y pueden manifestar emociones como la negación, que surge como una respuesta al dolor de tener un hijo con TEA, la culpa, el enajenamiento, la desconfianza y la incertidumbre respecto del futuro (Benjamín, 2006).

Cabe destacar, en relación al último punto, que en nuestra investigación el diagnóstico de los jóvenes, que fue en general a temprana edad (entre los 2 y 3 años en la mayoría de los casos), no significó un hecho en extremo doloroso, las madres de nuestro estudio señalan, que si bien en un comienzo fue duro, actuó como una especie de motor que las movilizó a ellas y sus familias a buscar alternativas que le brindaran mejores oportunidades a sus hijos.

Ahora bien, la literatura actual acerca del TEA, lo sitúa desde similares aristas a las que venimos abordando. Como es el caso de Cala, Licourt y Cabrera (2015), quienes en su trabajo realizado en Cuba, lo definen como una alteración del desarrollo caracterizada por deficiencias de tipo cualitativas en la comunicación e interacción social y un comportamiento que sigue patrones repetitivos y estereotipados, además de intereses restrictivos. Por su parte, Rico y Tárraga (2016), en España, conceptualizan el TEA como un trastorno del neuro-desarrollo que se caracteriza por déficit en la comunicación, interacción social y lenguaje, y además por la presencia de intereses restringidos y conductas estereotipadas, signos que se pueden identificar a temprana edad, y que afectarían al $1 \%$ de la población, siendo más frecuente en hombres que en mujeres en una 
proporción de 4:1. Por otro lado, el estudio de Díaz y Andrade (2015) sobre el TEA en la educación regular, realizado en instituciones educativas de Quito, Ecuador, deja en evidencia, que, al igual que en Chile, no existen cifras que den cuenta de la prevalencia del trastorno en el país.

En relación a las investigaciones que ponen el foco en la familia de personas con TEA, se destaca el estudio de Baña (2015), quien desarrolla en España una investigación que da cuenta que el rol de la familia es desde hace poco tiempo relevante, debido al modelo clínico-médico que según el autor aun predomina en el TEA, por sobre un modelo sistémico- social que brindaría gran importancia a la familia por ser referente en los primeros aprendizajes sociales y por su rol educador- estimulador. Siguiendo la temática familiar, García y Bustos (2015) señalan en su estudio desarrollado en México, que la discapacidad es un fenómeno complejo, una problemática multidimensional, y que por tanto, ninguna familia se encuentra preparada para hacer frente al problema en uno de sus miembros. Además, en España, Lozano y otros (2017), presentan una investigación donde se refieren a lo complejo que puede ser la recepción del diagnóstico del TEA para la familia, realizan una revisión de aspectos generales y proponen una intervención que beneficie a la familia e hijo con TEA.

En relación a estudios similares en Chile, la revisión realizada evidencias que no se han llevado a cabo investigaciones, que aborden la realidad de las familias de personas con TEA desde su propia voz.

\section{Experiencia vivida y construcción de representaciones sociales}

Como se mencionó anteriormente, esta investigación pretende visibilizar representaciones sociales a partir de los relatos de vida de 5 mujeres, cuya única relación y característica en común, es ser madre de un joven con trastorno del espectro autista.

Ahora bien, quien da carácter a la teoría de las representaciones sociales es Moscovici (1961), quien define las representaciones como sistemas cognitivos con una lógica y lenguaje propio para el descubrimiento y organización de la realidad. Es decir, plantea que las representaciones no son simples imágenes, sino que poseen capacidad de significación y la posibilidad de comunicación, lo cual pone de relieve el rol del lenguaje en su utilización. Posteriormente y desde la línea de Moscovici, Jodelet (1984) desarrolla su perspectiva de la teoría de las representaciones sociales y señala que han de ser comprendidas como la manera en que los sujetos sociales aprehenden los acontecimientos de la vida diaria, las características del medio ambiente y las informaciones insertas en este, a las personas del entorno próximo y lejano, las define como el conocimiento espontáneo, ingenuo, y que habitualmente es denominado conocimiento de sentido común. De esta conceptualización de Jodelet (1984) se destaca el concepto de aprehender, es decir, en este caso particular, cómo las madres toman, perciben o entienden lo que sucede en su vida diaria y en un aspecto específico, la experiencia educativa de sus hijos con TEA. Se verá en los resultados más adelante, la forma en que las madres toman y perciben las características del medio y las personas del entorno próximo, como lo son la respuesta de los centros educativos y de profesores y otros profesionales hacia sus hijos.

Por otro lado, González y otros (2009), y de manera similar a Jodelet (1984), conceptualizan las representaciones sociales como un conjunto de ideas, creencias y valores que tienen las personas en relación a sus temas de interés, y en general en relación a su forma de comprender el mundo. Es en este punto donde se conecta con el objeto de 
estudio, puesto que se pretende, a partir de los relatos de vida, rescatar el conjunto de ideas que tienen las madres de jóvenes con TEA, a partir de su forma de comprender el mundo respecto de cómo ha sido el proceso educativo de sus hijos. Es por tanto, a partir del relato de vida de las madres que se construye la experiencia educativa de los 5 jóvenes, todas y todos residentes en Concepción-Chile, desde donde emanan ideas, conceptos, valores y emociones sobre las diferentes situaciones de las que han sido parte, que llevan finalmente a la construcción de las representaciones sociales.

En este sentido, de acuerdo a Ricoeur (1985), en el relato de vida se presenta una doble interpretación, donde el investigador interpreta el relato de una persona, relato que a su vez, es una interpretación que la misma persona hace de su propia vida. Es decir, las madres al relatar en torno a la experiencia educativa de sus hijos, aun cuando es su propia vivencia hacen una interpretación de esta y los sucesos que fueron ocurriendo en el transcurso del tiempo. Es decir, el relato de las madres, la interpretación que ellas realizan de la experiencia de sus hijos, tienen un carácter temporal y contextual, que se relaciona con el ciclo vital de ellas y de los jóvenes, las vivencias, personas y situaciones, y circunstancias que los rodean actualmente, todo lo cual, sin duda, permite dar cuerpo a las representaciones sociales que se van construyendo.

Por lo tanto, las representaciones como conjunto de ideas, valores y formas de comprender el mundo, para esta investigación se abordan desde dos perspectivas. La primera, es en función de la relación de las madres con sus hijos, es decir, la forma en que ellas actúan y toman decisiones en base a la condición de los jóvenes. La segunda perspectiva que se propone, es en función de la percepción de las madres acerca de la experiencia de sus hijos en la sociedad, es decir, cómo la sociedad responde a la condición de sus hijos, específicamente en el ámbito educativo.

Dicho esto, ahora procedemos a rastrear la literatura empírica sobre el tema en el foco de las representaciones sociales. Aquí nos podemos encontrar a Duarte, Galindo y Sepúlveda (2019), quienes plasman representaciones sociales en un colegio de Cali-Colombia. Dichas representaciones se orientan hacia la discriminación de niños que presentan el diagnóstico. En Chile, Balbontín y Sklenar (2013) desarrollaron un trabajo sobre representaciones sociales en torno a la inclusión escolar en niños con TEA, en la comuna de San MiguelSantiago. En esta investigación, las autoras construyen las representaciones desde la voz de profesores, alumnos y apoderados. También cabe mencionar el estudio que realizaron Muñoz y Lazcano (2013), en un contexto similar al anterior, sobre representaciones sociales de la comunidad educativa en relación a los estudiantes del espectro autista y su inclusión escolar. Las autoras recurrieron a un establecimiento de Santiago, Chile, y develaron las representaciones desde la voz de la comunidad educativa. Por otro lado, de igual forma en Chile, Giaconi, Pedrero y San Martín (2017) llevaron a cabo un estudio sobre la discapacidad en niños y jóvenes. En este, plasman los significados que construyen los cuidadores en torno al concepto de discapacidad, y lo que implica ser cuidador de una persona que la presenta.

En suma, si bien se presentan estudios que abordan las representaciones sociales, estos no consideran directamente la percepción de madres y/o padres de niños o jóvenes con TEA en nuestro país. Sin embargo, se presenta un estudio llevado a cabo en Lima, Perú, de similares características a nuestra investigación. Dicha investigación fue desarrollada por Demos (2015) y se ocupa de representaciones del autismo en padres de hijos autistas. Para este estudio, se recurrió a 8 padres de jóvenes con el diagnóstico que asisten a una escuela 
especial, y se ahondó en temáticas como la representación de las dificultades y la representación del futuro del hijo autista.

\section{Método}

La investigación se enmarca en el paradigma interpretativo-cualitativo, caracterizado según Vain (2012, pp. 39-40) por tener un proceso de interpretación doble, donde se ponen en juego dos narrativas, en primer lugar las narraciones de un sujeto en relación a sus prácticas y discursos, y en segundo lugar las narraciones que realiza el investigador de acuerdo a lo que observa y a lo que los sujetos relatan. La primera interpretación por tanto, se refiere a la forma en que los sujetos interpretan su propia realidad, y la segunda, la forma en que el investigador intenta comprender el modo en que los sujetos construyen su realidad socialmente. Esta caracterización que hace Vain (2012), describe de manera clara y precisa la forma por la cual se pretenden construir las representaciones sociales.

A su vez, la investigación posee un enfoque fenomenológico, el cual según Creswell (1998, citado en Sandín, 2003, p. 151) "describe el significado de las experiencias vividas por una persona o grupo de personas acerca de un concepto o fenómeno". De acuerdo a esta conceptualización, la intención del estudio se refleja en este enfoque, puesto que se construyeron las representaciones sociales a partir del análisis de los relatos de vida, que reflejaban la experiencia educativa de los jóvenes desde la percepción de sus madres.

El relato de vida, utilizado como técnica de levantamiento de datos para llevar a cabo esta investigación, corresponde a una enunciación de parte de un narrador, ya sea de su vida o parte de esta y que puede ser oral o escrita (Cornejo, Mendoza y Rojas, 2008, p. 30). En este caso, el relato de vida corresponde a un relato oral de una parte específica de la vida de las madres, relacionada no únicamente con su vivencia, sino con la experiencia educativa de sus hijos, donde ellas juegan un rol activo y fundamental, en primer lugar por su calidad de madres, pero también por la condición de sus hijos, ya que debido a las características señaladas anteriormente, requerirán de cuidados y una atención especial y permanente. En cuanto al instrumento, se utilizó una pauta de entrevista para llevar a cabo el relato de vida de tipo semi- estructurado.

En relación a la muestra, esta fue de tipo selectiva o intencional, puesto que cada unidad fue seleccionada de forma cuidadosa e intencionada debido a la información detallada y profunda que podían ofrecer, sobre el tema de interés de la investigación (Martínez, 2012, pp. 614-615). La muestra quedó conformada por 5 mujeres, cuyos antecedentes se describen en el cuadro 1.

Cuadro 1. Antecedentes de participantes

\begin{tabular}{ccllc}
\hline MADRE & EDAD & NIVEL DE ESTUDIOS & \multicolumn{1}{c}{ OCUPACIÓN } & EDAD DE SU HIJO \\
\hline M1 & 52 años & Técnico profesional & Comerciante & 24 años \\
M2 & 52 años & Técnico profesional & Empleada pública & 24 años \\
M3 & 49 años & Universitario & Kinesióloga & 20 años \\
M4 & 47 años & $4^{\circ}$ medio & Dueña de casa & 19 años \\
M5 & 48 años & $4^{\circ}$ medio & Dueña de casa & 16 años \\
\hline
\end{tabular}

Fuente: Elaboración propia.

En cuanto al análisis de la información, se recurrió al procedimiento denominado análisis de contenido, utilizado con frecuencia para el análisis sistemático de notas de campo, entrevistas registradas, documentos escritos, memorias y otros, y que tiene por objetivo 
según Pérez-Serrano (2011, p. 134), estudiar de manera detallada el contenido de una comunicación oral, escrita o visual, tal como se hizo con los relatos de las madres participantes en el estudio.

El análisis de contenido llevó a la construcción de categorías de análisis, las cuales se presentan junto a sus respectivas subcategorías en la siguiente tabla. Cabe mencionar que para efectos de este artículo, únicamente se consideró la categoría la escuela y algunas subcategorías de la categoría expectativas.

Cuadro 2. Malla temática de análisis

\begin{tabular}{ll}
\hline \multicolumn{1}{c}{ TEMAS/ CATEGORÍAS } & \multicolumn{1}{c}{ SUBTEMAS/ SUBCATEGORÍAS } \\
\hline \multirow{2}{*}{ Diagnóstico } & Signos de alerta \\
& Proceso y especialistas \\
& Recepción del diagnóstico \\
& Conocimiento del TEA \\
\hline \multirow{3}{*}{ La escuela } & Primera experiencia educativa \\
& Valoración de la experiencia educativa en integración \\
& Valoración de la experiencia educativa en escuela especial \\
\hline \multirow{2}{*}{ Expectativas } & Futuro educativo \\
& Futuro laboral \\
& Redes de apoyo \\
& Temores \\
\hline
\end{tabular}

Fuente: Elaboración propia.

\section{Resultados}

Los relatos de las 5 madres permitieron finalmente la construcción de representaciones sociales en base a algunas de las categorías de análisis, las que se presentan a continuación en compañía de algunos extractos de los relatos:

\subsection{Representación social de la discriminación y del temor}

Se considera una representación social al existir confluencia en la decisión de las madres de enviar a sus hijos a un colegio de educación regular acompañados de una asistente particular. Por tanto, surge como una representación social de la discriminación y del temor, al señalar las madres de manera explícita que fue una decisión, incluso de pareja, que sus hijos asistieran a una escuela regular con una asistente, al no tener ellos la capacidad de defenderse en caso de que alguien les hiciera algo, asistente que tendría un rol activo como "la voz" de los jóvenes.

Como se menciona anteriormente, se presentan algunos extractos de los relatos de acuerdo a la categoría de análisis.

Categoría "La escuela"

Tres madres relatan que optaron por escuelas regulares para educar a sus hijos, pero siempre considerando el apoyo de una asistente personal que los acompañara en todo momento, frente a esto, se destacan los siguientes relatos:

(...) siempre fue opción para mí un colegio particular y que fuera con su asistente, porque nunca lo iba a mandar solo, o sea si lo mandaba solo prefería dejarlo en la 
casa, porque de todo lo que yo he visto eeeh, sabía de que iba a, una me daba susto, ya, porque él no se sabe defender. Madre de Vicente 1

Lo integramos pero con asistente, porque ellos no se saben defender, por cualquier cosa, siempre dependen de alguien, (...) sin la ayuda de la asistente no habríamos hecho nada, porque era ella la que se llevaba toda la pega pu (...) y no, si Jorge hubiese ido solo, habría sido un mueble (...), si ella cualquier cosa, era la voz del Jorge, era todo pu. Madre de Jorge

La madre de Agustín, de igual forma relata su decisión de contratar una asistente particular, sin embargo, no lo justifica a través del temor, más bien lo relata como una decisión de tipo práctica: “(...) tuvo una auxiliar por años, que iba de 8 a 8, todo el día con él, le preparaba todo, todo lo que había que hacer, lo que había que estudiar (...). Iba a clases con él”.

A partir de estos relatos, se puede concluir que si bien las madres (y las familias detrás de ellas) optaron por la opción de educación regular, el temor estuvo siempre latente, señalando incluso que sin la ayuda de la asistente, no habrían enviado a su hijo al colegio. El relato permite además concluir, que aun cuando optaron por escuelas regulares, estaban conscientes de que sus hijos solos en el colegio no hubieran tenido los mismos resultados:

Frente a esto la madre de Vicente señala: "Vice lo que avanzó, la verdad que lo avanzó gracias a ella no al colegio, el colegio no estaba ni ahí, mientras le pagaran era lo más importante".

En otro caso, la Madre de Antonio, quien optó por la escuela especial como primera opción, señala que no optó por una escuela regular porque para que resultara debía contratar una asistente particular, y eso significaba un costo económico: "(...) para ir a un colegio con integración tendría que ir con una asistente con algo (...), podría ir con una asistente, tienes que pagarle la asistente, cuanto te cobra una asistente".

Llama la atención esta idea de la necesidad de compañía y protección en la escuela regular, como un requisito fundamental para que los jóvenes (en ese entonces niños) pudieran asistir a sus escuelas, y en el caso de la madre de Antonio, surge la problemática económica que imposibilita que él asista a una escuela regular, pero finalmente esta cuestión está presente también en otros casos aquí analizados.

\subsection{Representación social de la no integración}

Las madres coinciden en su idea y valoración de la integración en escuelas regulares, señalando de manera enfática que esta no existe. Se considera una representación social puesto que corresponde al conocimiento de las madres adquirido a través de la experiencia, de su experiencia en las diferentes opciones educativas y en diferentes centros educativos, tal como señala Jodelet (1984), las madres expresan por medio de su representación el sentido que otorgan a su experiencia en el mundo social, y bajo este panorama, son enfáticas al señalar que la integración no existe, a causa de que no hay docentes ni profesionales preparados para dar respuesta a las necesidades de sus hijos, en general.

A continuación, algunos relatos que llevaron a la construcción de esta representación:

${ }^{1}$ Los nombres de los jóvenes han sido modificados para resguardar su identidad. 
Categoría "La escuela", subcategoría "Valoración de la experiencia educativa en integración"

Al relatar las madres sus experiencias en la escuela regular, se comienza a hacer evidente su postura frente a la integración que se genera en esta opción educativa, en este sentido, la Madre de Jorge señala:
Aaaaah...el colegio tenía las ganas no más, pero, no funciona, si no funciona la integración, porque los profes no están preparados, o sea cuando Jorge, primer y segundo año, como re bien (...) al final Jorgito estaba solamente en la sala de recursos especiales, ya no estaba con sus compañeros, y eso es lo que uno buscaba.

En el mismo sentido, la madre de Vicente relata:

La integración no funciona porque no hay funcionarios en el fondo, eeeh capaces de recibir estos niños no, no, no son gente realmente, eeeh que sea de corazón, no sé de profesión, yo creo que están ahí porque les pagan no más pu, puede que haya en algunas partes gente que se yo, pero lo que yo he visto, en general no.

La madre de Agustín por su parte, relata:

$A$ ver yo creo que la integración real sirve en una escuela eeeh normal hasta cierta edad (...) pero los profesores ya llegan un tiempo en que no quieren tener niños integrados po, yo no sé si porque les pagan poco, porque tienen muchos alumnos, mucho trabajo no tengo idea.

La madre de Antonio, aun cuando optó por la escuela especial, entrega en su relato su visión de la integración: "No existe, encuentro que no, no, a lo mejor para un niño Asperger podría ser, pero para un niño autista como el Antonio no, no, no existe la integración, no creo, que va aprender en un colegio, no (...)”.

Cabe destacar en estos relatos, que las madres coinciden en que las causas de que la integración no funcione se deben a la falta de preparación y a la poca disposición de los docentes, en contraposición a la opinión que tienen las madres acerca de los compañeros de curso de sus hijos, quienes en todos los casos fueron un factor positivo dentro del proceso educativo:

La madre de Pablo señala :“(...) los niñitos, como iba a una escuela normal los niñitos lo cuidaban harto... todos lo conocían, y como era un pueblo todos lo conocían”.

Por su parte, la madre de Vicente relata:

(...) los compañeros sobre todo en la escuela de Los Huertos, fueron súper empáticos con él, súper amorosos, lo cuidaban, andaban con la asistente y él, en ese sentido no, no hubo ni un problema (...), la cosa es que los niños se portaron súper bien con el Vicente, no tengo nada que decir.

Siguiendo la misma línea de los relatos anteriores, la madre de Jorge señala que

$$
\begin{aligned}
& \text { (...) los compañeros un } 7 \text {, o sea eso fue lo bueno, que desde chiquititos a los niños les } \\
& \text { empiezan a decir que hay niños diferentes, que hay que ayudarlos, que no tienen que } \\
& \text { tratarlos diferente, que es uno más. }
\end{aligned}
$$

Esto coincide con la experiencia de Agustín, donde su madre señala que son los docentes los que no están dados a trabajar con niños con algún diagnóstico y no así los compañeros: "Yo creo que más los profesores, los niños fíjate que no son discriminatorios".

\subsection{Representación social del futuro educativo negado}

Esta representación social se construye a partir del discurso de las madres que evidencia una gran decepción, molestia y preocupación por la falta de opciones educativas que tienen sus hijos luego de cumplir los 26 años, al finalizar su proceso de educación formal de 
acuerdo al Ministerio de Educación de Chile. Las madres confluyen en que existe por parte de los legisladores, falta de empatía y falta de conocimiento de la realidad que ellas y sus hijos viven, y bajo este panorama, no hay interés en mejorar esta situación en beneficio de los jóvenes con una discapacidad.

A continuación, extractos de algunos relatos que llevaron a la construcción de esta representación:

\title{
Categoría "expectativas", subcategoría "futuro educativo"
}

Las madres dentro del relato, señalan con actitud de decepción y a la vez molestia la situación que enfrentarán cuando sus hijos cumplan 26 años y culminen su proceso educativo formal.

\footnotetext{
Primero que nada, las leyes las encuentro súper estúpidas, porque, o sea a los 26 años me lo llevo para la casa y que no haga nada, porque es un niño que no va a poder trabajar, es un niño que no, que no va a poder valerse por si mismo, que depende absolutamente de mí, entonces me lo tengo que llevar para la casa según las leyes de este país pu. Madre de Vicente

(...) en el fondo nuestros hijos jubilan a los 26, eso pasa, ellos jubilan, y se jubilan y se quedan en la casa pu, y que vas a hacer con ellos en la casa, entretenerlos, enseñarles tú, eh, se van a aburrir. Madre de Antonio

(...) hemos visto casos, que han salido de la escuela por edad ¿y después que hacen?, se quedan en su casa no más sin hacer nada, entonces tampoco uno quiere ver eso. Madre de Jorge
}

La madre de Agustín por su parte, señala frente a esta situación, un factor que ella considera muy importante, el factor económico:

\begin{abstract}
To creo que más que el apoyo para el niño, debe haber un apoyo para los padres, porque el papá que no tiene educación, y el papá que no tiene recursos żqué hace con un niño a los 26 años en la casa?, si ellos tienen que salir a trabajar, por ejemplo, por ejemplo, entonces si esos papás tienen que salir a trabajar porque no tienen otra opción ¿Qué hacen con ese niño? ¿Dónde lo dejan? (...), porque qué pasa si en esta familia, tienen que salir a trabajar los dos j̇con quién dejan a este niño?, no sé una instancia de crear una guardería para adultos, ¿̇me entiendes?
\end{abstract}

Se aprecia de igual forma en los relatos, una sensación de invisibilización de la realidad que viven tanto los jóvenes como las familias de jóvenes con una discapacidad:

To no entiendo la persona que pensó esa ley que es hasta los 26, no sé qué pensó, la verdad, porque a mi me da la impresión de que las leyes se hacen solo para los niños que protestan, que marchan, que destrozan, todo eso, al tiro, pero estos son niños que no protestan, que no marchan, no gritan, no cierto, entonces nadie se ocupa de ellos, y nadie se, le interesa, como quien dice (...), total a nadie le importan estos niños. Madre de Vicente

To creo que no está resuelto el tema y no hay ni siquiera voluntad de resolver el tema porque no es un tema que les toque todavía, tendrían que tener un hijo para legislar. Madre de Agustín

A continuación, se presenta una tabla resumen con las representaciones sociales construidas a partir de los relatos de las madres pero desde dos perspectivas, primero, representaciones sociales de las madres a partir de la relación con sus hijos, y segundo, las mismas representaciones sociales pero desde la percepción de las madres sobre la experiencia de sus hijos en la sociedad, es decir, como la sociedad responde a la condición de su hijo con TEA. 
Cuadro 3. Resumen de representaciones sociales

\begin{tabular}{|c|c|}
\hline $\begin{array}{c}\text { REPRESENTACIONES SOCIALES DE LAS } \\
\text { MADRES A PARTIR DE LA RELACIÓN CON SUS } \\
\text { HIJOS }\end{array}$ & $\begin{array}{l}\text { REPRESENTACIONES SOCIALES DE LAS } \\
\text { MADRES A PARTIR DE SU PERCEPCIÓN DE LA } \\
\text { EXPERIENCIA DE SUS HIJOS EN LA SOCIEDAD }\end{array}$ \\
\hline $\begin{array}{l}\text { Representación social de la discriminación y } \\
\text { del temor: Las madres optan por una } \\
\text { escuela regular siempre con una asistente } \\
\text { particular, que acompañe a sus hijos } \\
\text { durante toda la jornada y que asuma como } \\
\text { "la voz" de los jóvenes por temor a que no } \\
\text { se puedan defender. }\end{array}$ & $\begin{array}{l}\text { Representación social de la discriminación y } \\
\text { del temor: La Sociedad (escuela regular) no } \\
\text { estaría preparada para brindar la atención y } \\
\text { cuidados necesarios a los jóvenes con TEA. } \\
\text { Profesores o compañeros podrían tener } \\
\text { conductas inadecuadas y ellos no sabrán } \\
\text { defenderse. }\end{array}$ \\
\hline $\begin{array}{l}\text { Representación social de la no integración: } \\
\text { Madres manifiestan que desde su } \\
\text { perspectiva la integración no existe, no } \\
\text { existen los profesionales capaces de hacerse } \\
\text { cargo realmente de los estudiantes con este } \\
\text { diagnóstico. }\end{array}$ & $\begin{array}{l}\text { Representación social de la no integración: La } \\
\text { escuela regular retira a los estudiantes de la } \\
\text { sala común, a la sala de recursos especiales } \\
\text { cada vez con mayor frecuencia, no les } \\
\text { permite compartir con el grupo curso. } \\
\text { Finalmente es la escuela regular quien } \\
\text { recomienda buscar otras opciones } \\
\text { educativas para los jóvenes. }\end{array}$ \\
\hline $\begin{array}{l}\text { Representación social del futuro educativo } \\
\text { negado: } \\
\text { Las madres señalan con molestia y decepción } \\
\text { que sus hijos no tiene acceso a sistemas de } \\
\text { educación posterior a los } 26 \text { años. }\end{array}$ & $\begin{array}{l}\text { Representación social del futuro educativo } \\
\text { negado: } \\
\text { La Sociedad niega la posibilidad de educación } \\
\text { a jóvenes con TEA (u otra condición) } \\
\text { posterior a los } 26 \text { años, a causa de una ley } \\
\text { que así lo establece. } \\
\text { No se entregan las posibilidades necesarias ni } \\
\text { las bases legales, para que los jóvenes } \\
\text { continúen escolarizados o en alguna otra } \\
\text { instancia formativa. }\end{array}$ \\
\hline
\end{tabular}

Fuente: Elaboración propia.

\section{Conclusiones}

Las representaciones sociales (Moscovici, 1961; Jodelet, 1984) de la experiencia educativa de jóvenes con TEA, fueron construidas mediante el análisis categorial de los relatos de vida de las madres bajo un enfoque fenomenológico (Husserl, 1990), es decir, considerando siempre la experiencia tal como fue vivida y percibida por sus protagonistas. Fue en el transcurso del relato que comenzaron a emanar las ideas, valores y emociones en relación a la experiencia educativa. De igual forma, comenzaron a surgir términos, ideas, experiencias y emociones en común, que permitieron finalmente comprender la realidad tal como fue vivida por las madres, interpretando y representando el sentir de sus hijos.

En relación al primer objetivo específico, los relatos de las madres, señalan que cuatro de ellos asistieron a establecimientos de educación regular como primera opción y todos ellos, además, asistieron a temprana edad a jardines infantiles y escuela de lenguaje. Cabe destacar que en tres casos, los estudiantes asistieron a una escuela regular con la compañía permanente de una asistente particular (costeada por los padres). El quinto estudiante en cambio, asistió a escuela especial como primera opción poco después de cumplir los 3 años (edad en que fue diagnosticado). En este caso, la madre señala en su relato estar conforme con su decisión y manifestó su interés en que su hijo se mantenga en esta opción educativa.

A partir del punto anterior, es posible dar respuesta al segundo objetivo específico, puesto que surge la primera emoción en el relato de las madres, se evidencia un gran temor a dejar a sus hijos solos en la escuela, temor que se fundamenta en que alguien podría dañar a sus hijos y ellos no sabrían defenderse, por tanto la asistente particular cumplía el rol de 
"voz" de los estudiantes. En los cinco casos, las madres optaron finalmente por la opción de escuela especial, y de acuerdo a sus relatos, tienen la intención de continuar en esta opción educativa puesto que cumple con sus expectativas, perciben que sus hijos se sienten cómodos y que les agrada asistir y por tanto la consideran una experiencia positiva.

En este sentido, cobra relevancia el concepto de representación social de Jodelet (1984), quien lo define como la manera en que las personas aprehenden las características del medio ambiente y las informaciones que están insertas en éste, así, las madres visualizan y perciben el ambiente inmediato de sus hijos, es decir, la escuela regular, como un lugar inseguro, donde sus hijos no pueden desenvolverse de manera libre e independiente, y donde incluso podrían vivir situaciones de violencia o vulneración.

En la actualidad (año 2019), los cinco estudiantes se encuentran matriculados en escuelas especiales, lugar donde pueden asistir hasta los 26 años de edad de acuerdo a la legislación del Ministerio de Educación en Chile. Es en este contexto donde el relato de las madres evidencia otras emociones, decepción y molestia, puesto que sienten que el sistema educativo les priva la oportunidad de continuar asistiendo a sus escuelas. En relación a lo anterior, las madres manifiestan un cierto temor hacia el futuro, señalando que prefieren no pensar en qué pasará más adelante y solo vivir el presente.

En cuanto a las representaciones sociales visibilizadas y construidas a través de este estudio, se encuentra la representación social de la discriminación y del temor, representación social de la no integración y la representación social del futuro educativo negado, cada una de las cuales fueron descritas en el apartado anterior.

Entendiendo que la escuela no solo brinda aprendizajes de contenido, sino también les permite a los jóvenes aprender habilidades para la vida diaria, construir relaciones sociales y afectivas y, en algunos casos, adquirir herramientas para la inserción laboral, se puede proyectar a partir de las representaciones sociales construidas con los relatos de las madres, mencionados anteriormente, la opción de ahondar en próximas investigaciones, sobre el futuro ocupacional de personas con discapacidad luego de culminar su proceso educativo. Esto, debido a que se evidenció en los relatos un gran temor hacia ese periodo de la vida de los jóvenes, visualizando la respuesta de la sociedad como una negación hacia el derecho de sus hijos a recibir educación.

También, se señala como una limitación para este estudio, el hecho de considerar solo a estudiantes hombres y no mujeres. Lo anterior se debe únicamente a la prevalencia del diagnóstico, el cual se presenta en una mujer por cada cuatro hombres (MINSAL, 2011), como ya ha sido mencionado. Debido a lo anterior, fue difícil encontrar a estudiantes mujeres, y en los casos contactados, no fue posible establecer vínculo para la participación, por tanto la muestra quedó constituida únicamente por hombres con TEA representados por el relato de sus madres.

Al finalizar el estudio, pareciera poco alentador el futuro educativo y ocupacional de personas con discapacidad, puesto que la legislación actual en Chile, en lugar de brindar la oportunidad de seguir adquiriendo habilidades y disfrutando de espacios sociales, los limita a ellos y a sus familias, quienes se ven obligados a dejarlos en la casa sin las posibilidades que brinda la escuela. En este sentido, se concluye que el sistema educativo no está brindando la respuesta que las familias y especialmente los estudiantes con TEA u otra condición requieren en Chile. 


\section{Referencias}

American Psychiatric Association. (2013). Manual diagnóstico y estadístico de los trastornos mentales $(D S M)$. Madrid: Editorial Médica Panamericana.

Arce, M. (2008). Autismo. Buenos Aires: Landeira.

Balbontín, P. y Sklenar, D. (2013). Las representaciones sociales en torno a la inclusión escolar en niños con trastorno del espectro autista: una visión al proyecto de integración escolar en un colegio de la comuna de San Miguel (Tesis de pregrado). Santiago de Chile: Universidad Andrés Bello.

Baña, M. (2015). El rol de la familia en la calidad de vida y la autodeterminación de las personas con trastorno del espectro del autismo. Ciencias Psicológicas, 9(2), 323-336.

Benjamín, B. (2006). Un niño especial en la familia. Guía para padres. Ciudad de México: Trillas.

Blanco, R. (2006). La equidad y la inclusión social: Uno de los desafíos de la educación y la escuela hoy. REICE. Revista Iberoamericana sobre Calidad, Eficacia y Cambio en Educación, 4(3), 1-15.

Borax, J. y Buron, V. (2017). Trastorno del espectro autista. Recuperado de https://www.camara.cl/

Cala, O., Licourt, D. y Cabrera, N. (2015). Autismo. Un acercamiento hacia el diagnóstico y la genética. Revista de Ciencias Médicas, 19(1), 157-178.

Cornejo, M., Mendoza, F. y Rojas R. (2008). La investigación con relatos de vida: Pistas y opciones del diseño metodológico. PSTKHE, 17(1), 29-39.

https://doi.org/10.4067/So7 18-22282008000100004

Díaz, E. y Andrade, I. (2015). El trastorno del espectro autista (TEA) en la educación regular: Estudio realizado en instituciones educativas de Quito, Ecuador. Revista Intercontinental de Psicología y Educación, 17(1), 163-181.

Delfos, M. F. (2006). A strange world-autism, asperger's syndrome and pdd-nos. Philadelphia, PA: Jessica Kingsley Publishers.

Demos, A. (2015). Representaciones del autismo en padres de hijos autistas (Tesis de pregrado). Lima: Pontificia Universidad Católica Del Perú.

Duarte, R., Galindo, L. y Sepúlveda M. (2019). Representaciones sociales sobre el autismo en un grupo de docentes de primaria del colegio Luis Madina de Cali. (Tesis de pregrado). Cali: Fundación Universitaria Católica Lumen Gentium.

García, R. y Bustos, G. (2015). Discapacidad y problemática familiar. Paakat: Revista de Tecnología y Sociedad, 5(8). art 5 .

Giaconi, C., Pedrero, Z. y San Martín, P. (2017). La discapacidad: Percepciones de cuidadores de niños, niñas y jóvenes en situación discapacidad. Psicopespectivas, 16(1), 55-66. https://doi.org/10.5027/psicoperspectivas-Vol16-Issue1-fulltext-822

González, F., Rivera, M., Galindo, M. y Grandón, O. (2009). Representaciones sociales sobre compresión y producción de textos. Ciudad de México: Universidad de Sonora.

Hernández, V., Calixto, B. y Aguilar, I. (2012). Aspectos psicológicos de familiares de personas diagnosticadas con trastorno del espectro autista. Revista Intercontinental de Psicología y Educación, 14(1), 73-90.

Husserl, E. (1990). Artículo de la Enciclopedia Británica. Ciudad de México: UNAM.

Jodelet, D. (1984). La representación social. Fenómenos, concepto y teoría. En S. Moscovici (Ed.), Psicología social II. Psicología social y problemas sociales (pp. 469-494). Barcelona: Paidós. 
Lambert, M. (2018). Trastorno del espectro autista. Epidemiología, aspectos psicosociales, y políticas de apoyo en Chile, España y Reino Unido. Recuperado de https://www.bcn.cl/obtienearchivo?id=repositorio/10221/25819/1/BCN_Politicas_de_ apoyo_al_espectro_autista_FINAL.pdf

Lozano, M., Manzano, A., Casiano, C. y Aguilera, C. (2017). Propuesta de intervención en familiares de niños con TEA desde ACT para mejorar la convivencia familiar y escolar. INFAD Revista de Psicología, 1(1), 45-56. https://doi.org/10.17060/ijodaep.2017.n1.v1.897

Martínez, C. (2012). El muestreo en investigación cualitativa. Principios básicos y algunas controversias. Ciencia \&̊ Saudé Colectiva, $17(3), 613-619$. https://doi.org/10.1590/S1413-81232012000300006

Mesibov, G. y Howley, M. (2010). El acceso al curriculum por alumnos con trastornos del espectro del autismo. Madrid: Autismo Ávila.

MINEDUC. (2005). Política nacional de educación especial, nuestro compromiso con la diversidad. Santiago de Chile: MINEDUC.

MINEDUC. (2009). Decreto 170: Fija normas para determinar los alumnos con necesidades educativas especiales que serán beneficiarios de las subvenciones para educación especial. Santiago de Chile: MINEDUC.

MINEDUC. (2015). Propuestas para avanzar hacia un sistema inclusivo en Chile: Un aporte desde la educación especial. Santiago de Chile: MINEDUC.

MINSAL. (2011). Detección temprana de los trastornos del espectro autista TEA. Santiago de Chile: MINSAL.

Morán, D. (2011). Introducción a la fenomenología. Barcelona: Anthropos.

Moscovici, S. (1961). El psicoanálisis, su imagen y su público. Buenos Aires: Huemul.

Muñoz A. y Lazcano, V. (2013). Representaciones sociales de la comunidad educativa en relación a los estudiantes con trastorno del espectro autista y su inclusión escolar (Tesis de pregrado). Santiago de Chile: Universidad Andrés Bello.

Organización Mundial de la Salud. (2017). Trastornos del espectro autista. Recuperado de https://www.who.int/es/news-room/fact-sheets/detail/autism-spectrum-disorders

Pérez-Serrano, G. (2011). Investigación cualitativa. Retos e interrogantes. Madrid: La Muralla.

Rico, J. y Tárraga, R. (2016). Comorbilidad de TEA y TDAH: Revisión sistemática de los avances en investigación. Anales de psicología, 32(3), 810-819.

https://doi.org/10.6018/analesps.32.3.217031

Ricœur, P. (1985). Temps et récit. París: Editions du Seuil.

Sandín, M. P. (2003). Investigación cualitativa en educación. Fundamentos y tradiciones. Madrid: Mc Graw Hill.

SENADIS. (2016). Estudio nacional de la discapacidad. Santiago de Chile: SENADIS.

Taylor, R., Smiley, L. y Richards, S. (2009). Estudiantes excepcionales. Formación de maestros para el siglo XXI. Ciudad de México: Mc Graw Hill.

Vain, D. (2012). El enfoque interpretativo en investigación educativa: Algunas consideraciones teórico-metodológicas. Revista de Educación, 3(4), 37-46.

Wing, L. (1998). El autismo en niños y adultos. Una guía para la familia. Barcelona: Paidós. 


\section{Breve CV de los autores}

\section{Camila Andrea Toledo Manríquez}

Profesora de Educación Diferencial mención Deficiencia Mental, Licenciada en Educación de la Universidad de Concepción, finalizó sus estudios en enero de 2016. Un año después, en marzo de 2017, ingresó al programa de Magíster en psicopedagogía y Educación Especial en la Universidad Católica de la Santísima Concepción, marco formativo en que se desarrolla el presente artículo y de cuyo programa ya egresó. Actualmente se desempeña como docente en el Centro de Capacitación Laboral de Educación Especial Paulo Freire, ubicado en Concepción. ORCID ID: https://orcid.org/0000-0001-85523705. Email: camilatoledomanriquez@gmail.com

\section{Óscar Fernando Basulto Gallegos}

Licenciado en Comunicación Social, Magíster en Comunicación Estratégica y Doctor en Sociología. Forma parte del claustro académico del programa de Magíster en psicopedagogía y educación especial en la Universidad Católica de la Santísima Concepción y está a cargo de la asignatura Taller de análisis de datos cualitativos, y dirige seminarios de investigación en el mismo programa. Además, se encuentra adscrito como académico a la Facultad de Comunicación, Historia y Ciencias Sociales de la misma universidad. ORCID ID: https://orcid.org/0000-0001-8323-1098. Email: obasulto@ucsc.cl 